\title{
Medical genetics: advances in brief
}

Psychological impact of populationbased carrier testing for cystic fibrosis: 3-year follow up

Axworthy D, Brock DJH, Bobrow M, Marteau TM, for the UK Cystic Fibrosis Follow-up Study Group. Lancet 1996;347:1443-6.

Commentary. Whole population screening for carriers of cystic fibrosis gene Carswell F. Lancet 1996;347:1421-2.

Axworthy et al attempt to find out the long term consequences of population screening for cystic fibrosis carriers in terms of understanding of results, reproductive behaviour and intentions, anxiety, and perception of health, three years after testing. Questionnaires were sent to 435 identified carriers from a total population of 13863 people screened. For each carrier two age and sex matched controls who had received screen negative results were chosen. The sample was selected from six projects that had investigated uptake and short term consequences of cystic fibrosis screening in the UK. Three of the studies had offered testing to the general population through primary care physicians and three had offered testing to unselected samples of pregnant parents. Sixty two percent of questionnaires were returned, with a slightly higher proportion being returned from carriers $(68 \%)$ than from screen negative subjects $(59 \%) ; 81 \%$ of carriers correctly remembered their result although $16 \%$ believed only that they were likely to be carriers. More worryingly $50 \%$ of screen negative people believed that they were definitely not carriers. The proportion of those who were screen negative who correctly understood their results had fallen from high levels soon after testing as documented in the earlier reports. Anxiety was not shown to be different between carriers and screen negative subjects, but carriers did perceive their current health more poorly (although not their past or future health). There were no differences between the groups in their reproductive behaviour or intentions. The response rate to the questionnaires was influenced by the result received, the centre where testing was carried out, and age. The authors suggest that poor recall of results is caused by psychological mechanisms to simplify and reduce risk perception. However, since accurate recall does not necessarily influence behaviour to reduce risk they suggest that improving recall of screening results should not be the only goal of testing programmes. Since the minimisation of risk perception may help people to cope with negative emotions, promoting accurate recall could interfere with this protective process. In his commentary Dr Carswell suggests that neonatal screening for cystic fibrosis may have more influence on subsequent parental behaviour. The value of population based screening for cystic fibrosis is the ability to detect at risk partnerships without previous family history, but further research to increase its efficacy in promoting accurate understanding of results and informed reproductive decisions is needed before its generalised acceptance.

\section{ANGELA BARNICOAT}

Neuroblastoma and congenital cardiovascular malformations

Rosti L, Lin AE, Frigiola A. Pediatrics 1996;97:258-60.

Neuroblastoma is the most common malignancy in the first year of life $(7-10 \%$ of childhood tumours), presenting as both clinically apparent tumours or clinically unsuspected in situ tumours detected at necropsy. Some tumours regress spontaneously, making it difficult to determine the true frequency of neuroblastoma, which is estimated at about 6-10/100 000 children. A recent report suggests that there may be a neuroblastoma tumour suppressor locus at $1 \mathrm{p} 36$. The authors of this paper draw attention to a possible association of neuroblastoma with congenital cardiovascular malformations, especially conotruncal defects, and report a boy with a prenatally detected congenital heart defect and congenital neuroblastoma. They review the published findings in 19 patients who had congenital heart disease and neuroblastoma and mention a previous study in which $11.1 \%$ of neuroblastoma patients were found to have congenital heart defects compared to a $4.8 \%$ incidence of congenital cardiac defects in general necropsy patients. The authors discuss a classification system for congenital cardiac defects based on the possible embryological mechanisms and suggest that there is an apparent increase of conotruncal cardiac malformations in the patients with neuroblastoma. They speculate that abnormal neural crest cell development and migration may explain the coexistence of neuroblastoma and conotruncal heart defects. One other possibility is that chronic hypoxaemia postnatally might stimulate neural crest derived cells to proliferate. The authors used molecular studies to rule out both a 1 p36 deletion and a 22q11 deletion in the child they reported, because of the association of conotruncal heart defects with the 22q11 locus. Given that this is a retrospective study on a small number of case reports, there may well be an ascertainment bias. However, the occurrence of specific conotruncal defects such as right sided aortic arch and vascular rings within this study group does lend some weight to the authors' hypothesis. Work going on to isolate genes within the $22 \mathrm{q} 11$ and the $1 \mathrm{p} 36$ regions may help to clarify the situation in due course.

\section{JILL CLAYTON-SMITH}

Differential effects of FGFR2 mutations on syndactyly and cleft palate in Apert syndrome

Slaney SF, Oldridge M, Hurst JA, et al. Am $\mathcal{F}$ Hum Genet 1996;58:923-32.

Apert syndrome (acrocephalosyndactyly) is an autosomal dominant malformation characterised by craniosynostosis and severe symmetrical syndactyly of the hands and feet. The birth prevalence is about 1 in 65000 , and most cases arise as new mutations, with a clear association with raised paternal age. It is caused by specific missense substitutions involving adjacent amino acids (Ser252Trp or Pro253Arg) in the linker between the second and third extracellular immunoglobulin domains of fibroblast growth factor receptor 2 (FGFR2). In this study, the DNA from 70 unrelated patients with Apert syndrome was analysed using a simple PCR assay for these mutations in genomic DNA, based on the creation of novel $S f i$ and $B s t \mathrm{UI}$ restriction sites. Forty-five patients had the Ser252Trp mutation, and 25 the Pro253Arg mutation. Phenotypic differences between the two groups were investigated and significant differences emerged for the severity of syndactyly and the presence of cleft palate. Cleft palate was more common in patients with the former mutation, while syndactyly was more severe with the latter. Other genotype/phenotype correlations did not reach statistical significance. The opposite trends for severity of syndactyly and cleft palate in relation to the two mutations may relate to the varying patterns of temporal and tissue specific expression of different fibroblast growth factors, the ligands for FGFR2. Meanwhile, mutations in other regions of the FGFR2 gene have been associated with the Pfeiffer and Jackson-Weiss craniosynostosis syndromes, in which the limb mutations are milder than in Apert syndrome, and with Crouzon syndrome, in which the limbs are usually normal. With these latter three craniosynostosis syndromes, in some cases the same mutation has been associated with more than one syndrome, whereas the two mutations responsible for Apert syndrome are very specific.

FRANCES FLINTER 